\title{
Uniform decay for the coupled Klein-Gordon-Schrödinger equations with locally distributed damping
}

\author{
V. BISOGNIN* \\ Department of Mathematics, UNIFRA \\ 97010-032, Rua dos Andradas 1614 \\ Santa Maria, RS, Brazil \\ e-mail:vanilde@unifra.br
}

\author{
M. M. CAVALCANTI ${ }^{\dagger}$, V. N. DOMINGOS CAVALCANTI ${ }^{\ddagger}$, \\ J. SORIANO ${ }^{\S}$ \\ Department of Mathematics, State University of Maringá \\ 87020-900, Av. Colombo 5790 \\ Maringá, PR, Brazil \\ e-mail: mmcavalcanti@uem.br \\ e-mail: vndcavalcanti@uem.br \\ e-mail: jaspalomino@uem.br
}

\begin{abstract}
The following coupled damped Klein-Gordon-Schrödinger equations are considered

$$
\begin{aligned}
i \psi_{t}+\Delta \psi+i \alpha|\psi|^{2} \psi & =\phi \psi \text { in } \Omega \times(0, \infty),(\alpha>0) \\
\phi_{t t}-\Delta \phi+a(x) \phi_{t} & =|\psi|^{2} \chi_{\omega} \text { in } \Omega \times(0, \infty),
\end{aligned}
$$

where $\Omega$ is a bounded domain of $\mathbb{R}^{n}, n \leq 3$, with smooth boundary $\Gamma$ and $\omega$ is a neibourhood of $\partial \Omega$. Here $\chi_{\omega}$ represents the characteristic function of $\omega$. Assuming that $a \in W^{1, \infty}(\Omega)$ is a nonnegative function such that $a(x) \geq$ $a_{0}>0$ a. e. in $\omega$, polynomial decay rate is proved for every regular solution of the above system. Our result generalizes substantially the previous results given by the authors in the reference $[\mathrm{CDC}]$.
\end{abstract}

\footnotetext{
${ }^{\dagger}$ Research of M. M. C. partially supported by the CNPq Grant 300631/2003-0

${ }^{\ddagger}$ Research of V. N. D. C. partially supported by the CNPq Grant $304895 / 2003-2$

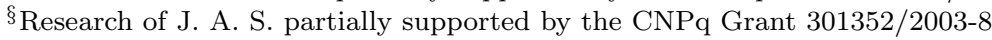


2000 Mathematics Subject Classification: 35L70, 35B40.

Key words: Klein-Gordon-Schrödinger, Localized damping.

\section{Introduction}

We consider the following model of Klein-Gordon-Schrödinger equations with locally distributed damping

$$
\left\{\begin{array}{l}
i \psi_{t}+\Delta \psi+i \alpha|\psi|^{2} \psi=\phi \psi \text { in } \Omega \times(0, \infty) \\
\phi_{t t}-\Delta \phi+a(x) \phi_{t}=|\psi|^{2} \chi_{\omega} \text { in } \Omega \times(0, \infty) \\
\psi=\phi=0 \text { on } \Gamma \times(0, \infty) \\
\psi(0)=\psi_{0} \in H_{0}^{1}(\Omega) \cap H^{2}(\Omega), \\
\phi(0)=\phi_{0} \in H_{0}^{1}(\Omega) \cap H^{2}(\Omega), \\
\phi_{t}(0)=\phi_{1} \in H_{0}^{1}(\Omega),
\end{array}\right.
$$

where $\Omega$ is a bounded domain of $\mathbb{R}^{n}, n \leq 3$, with smooth boundary $\Gamma$ and $\omega$ is an open subset of $\Omega$ such that meas $(\omega)>0$. In what follows, $\alpha$ is a positive constant and $\chi_{\omega}$ represents the characteristic function, that is, $\chi=1$ in $\omega$ and $\chi=0$ in $\Omega \backslash \omega$. We consider $a \in W^{1, \infty}(\Omega)$ a nonnegative function such that

$$
a(x) \geq a_{0}>0 \text { a.e. in } \omega,
$$

so that the nonlinearity $|\psi|^{2}$ exists where the damping $a(x) \phi_{t}$ is, in fact, effective and reciprocally. If the damping is effective in the whole domain, i. e., $a(x) \geq$ $a_{0}>0$ a. e. in $\Omega$ we can consider $\chi_{\omega} \equiv 1$ in $\Omega$. This is required in order to turn the system dissipative. Problem (1.1) has its origin in the canonical model of the Yukawa interaction of conserved complex nucleon field $\psi$ with neutral real meson field $\phi$ given by

$$
\left\{\begin{array}{l}
i \psi_{t}+\Delta \psi=\phi \psi \text { in } \Omega \times(0, \infty) \\
\phi_{t t}-\Delta \phi+\mu^{2} \phi=|\psi|^{2} \text { in } \Omega \times(0, \infty) \\
\psi=\phi=0 \text { on } \Gamma \times(0, \infty) \\
\psi(0)=\psi_{0}, \phi(0)=\phi_{0}, \phi_{t}(0)=\phi_{1} .
\end{array}\right.
$$

Where the positive constant $\mu$ represents the mass of a meson. Since we are considering a bounded domain, for simplicity's sale the term $\mu^{2} \phi$ will be omitted.

It is important to note that problem (1.2) is not naturally dissipative. So, the introduction of the dissipative mechanisms given by the terms $\alpha|\psi|^{2} \psi$ and $a(x) \phi_{t}$ are necessary to force the energy to decay to zero when $t$ goes to infinity. In fact, the dissipative K-G-S equation has been widely studied, see for example the following references: [BoYo1], [BoYo3], [LaWa1], [LaWa2], [MoGoHa], [GaDa] and references therein. The majority of works in the literature deal with linear dissipative terms acting in both equations, except for the works [BoYo2] and $[\mathrm{CDC}]$. Very few is known, in terms of polynomial decay, regarding the natural 
nonlinear dissipation $i|\psi|^{2} \psi$ acting in the Schrödinger equation and, as far as we are concerned, there is no result in the literature dealing with a localized dissipation in the wave equation for this system. A natural question arises in this context: it would be possible to consider a localized feedback $i b(x)|\psi|^{2} \psi$ acting in the Schrödinger equation (instead of a mechanism of damping acting on the whole domain) in order to obtain some decay rate? This is a hard open problem to be solved since some 'good terms' are lost in the computations when we consider the real (or imaginary) part of them. In the present paper, since we are considering a nonlinear feedback acting in the Schrödinger equation and a linear localized one acting in the wave equation, it is expected that the energy of the system decays to zero polynomially. To prove this fact is the main goal of this paper.

We would like to mention other papers in connection with problem (1.2) namely: Fukuda and Tsutsumi [FT1], [FT2], [FT3], [FT4], Bachelot and Chadam [BaCha] and Hayashi and W. Von Wahl [HaVo]. In the above articles the unique global existence to problem (1.2) is established and some conservation laws are verified.

The strategy to prove polynomial and uniform decay rates to problem (1.1) is to obtain integral inequalities of energy. For this purpose we have to use the multiplier method due to L. F. Ho [Ho], which is detailed in Lions [Li2], combined with integral inequalities that can be found in Komornik [K], (and references therein) with new tools which come from the difficulty in dealing with this type of coupled equations. Our result generalizes substantially the previous results due to the authors Cavalcanti and Domingos Cavalcanti given in [CDC]. In [CDC] the mechanism of damping is effective in the whole domain for both equations.

Our paper is organized as follows. In section 2 we give the precise assumptions and state our main result and in section 3 we give the proof of the main theorem.

\section{Main Result}

In what follows let us consider the Hilbert space $L^{2}(\Omega)$ of complex valued functions on $\Omega$ endowed with the inner product

$$
(u, v)=\int_{\Omega} u(x) \overline{v(x)} d x,
$$

and the corresponding norm

$$
\|u\|_{2}^{2}=(u, u) .
$$

We also consider the Sobolev space $H^{1}(\Omega)$ endowed with the scalar product

$$
(u, v)_{H^{1}(\Omega)}=(u, v)+(\nabla u, \nabla v) .
$$

We define the subspace of $H^{1}(\Omega)$, denoted by $H_{0}^{1}(\Omega)$, as the closure of $C_{0}^{\infty}(\Omega)$ in the strong topology of $H^{1}(\Omega)$. This space endowed with the norm induced by the 
scalar product

$$
(u, v)_{H_{0}^{1}(\Omega)}=(\nabla u, \nabla v)
$$

is, thanks to the Poincaré's inequality

$$
\|u\|_{2} \leq \lambda\|\nabla u\|_{2}, \quad \text { for all } u \in H_{0}^{1}(\Omega)
$$

a Hilbert space. We set the norms

$$
\|u\|_{p}^{p}=\int_{\Omega}|u(x)|^{p} d x, \quad\|u\|_{\Gamma, p}^{p}=\int_{\Gamma}|u(x)|^{p} d \Gamma, \quad\|u\|_{\infty}=\operatorname{ess}_{\sup } \in \Omega(u(x) \mid .
$$

In the particular case when $p=4$ we have the continuous immersion $L^{4}(\Omega) \hookrightarrow L^{2}(\Omega)$ and consequently the following inequality holds

$$
\|v\|_{2} \leq k\|v\|_{4}, \quad \text { for all } v \in L^{4}(\Omega),
$$

where $k:=\operatorname{meas}(\Omega)^{1 / 4}$.

The following assumptions are made:

Assumption 2.1 We assume that $a \in W^{1, \infty}(\Omega)$ is a nonnegative function such that

$$
a(x) \geq a_{0}>0, \quad \text { a. e. in } \omega .
$$

In addition,

$$
\text { If } a(x) \geq a_{0}>0 \text { in } \Omega \text {, then we consider } \chi_{\omega} \equiv 1 \text { in } \Omega \text {. }
$$

Assumption 2.2 We assume that $\omega$ is a neighbourhood of $\overline{\Gamma\left(x^{0}\right)}$ where

$$
\Gamma\left(x_{0}\right):=\left\{x \in \Gamma ;\left(x-x^{0}\right) \cdot \nu(x)>0\right\}
$$

and $\nu(x)$ is the unit outward normal at $x \in \Gamma$.

As an example of a domain $\Omega$ satisfying the above assumption let us consider the figure 1 below: 


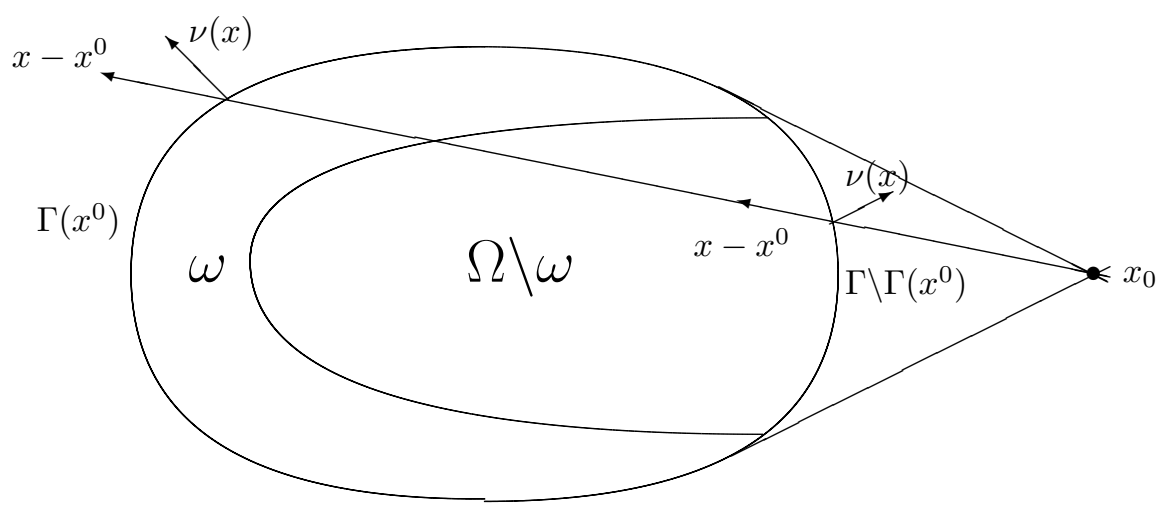

Figure 1

It is well known that under the Assumption 2.1, problem (1.1) is well posed in the space $H_{0}^{1}(\Omega) \cap H^{2}(\Omega) \times H_{0}^{1}(\Omega) \cap H^{2}(\Omega) \times H_{0}^{1}(\Omega)$, that is for any initial data $\left\{\psi_{0}, \phi_{0}, \phi_{1}\right\} \in H_{0}^{1}(\Omega) \cap H^{2}(\Omega) \times H_{0}^{1}(\Omega) \cap H^{2}(\Omega) \times H_{0}^{1}(\Omega)$ there exists a unique regular solution of (1.1) in the class

$$
\begin{aligned}
& \psi \in L_{l o c}^{\infty}\left(0, \infty, H_{0}^{1}(\Omega) \cap H^{2}(\Omega)\right), \phi \in L_{l o c}^{\infty}\left(0, \infty, H_{0}^{1}(\Omega) \cap H^{2}(\Omega)\right), \\
& \phi_{t} \in L_{l o c}^{\infty}\left(0, \infty, H_{0}^{1}((\Omega)) .\right.
\end{aligned}
$$

The energy associated to problem (1.1) is defined by

$$
E(t):=\frac{1}{2} \int_{\Omega}\left(|\psi(x, t)|^{2}+|\nabla \phi(x, t)|^{2}+\left|\phi_{t}(x, t)\right|^{2}\right) d x .
$$

Now, we are in position to state our main result

Theorem 2.1 Assume that assumptions (2.1) and (2.2) hold and moreover that $\alpha>\frac{a_{0}^{-1}}{2}$. Then, there exists some positive constant $C=C(E(0))$ such that following decay rate holds

$$
E(t) \leq \frac{C E(0)}{1+t}, \quad \text { for all } t \geq 0
$$

for every regular solution of problem (1.1) in the class given in (2.6)

\section{Uniform Decay Rates}

In this section we work it regular solutions $\left\{\psi(t), \phi(t), \phi_{t}(t)\right\}$ to problem (1.1), that is, those ones that lie, for instance, in $H_{0}^{1}(\Omega) \cap H^{2}(\Omega) \times H_{0}^{1}(\Omega) \cap H^{2}(\Omega) \times H_{0}^{1}(\Omega)$. In what follows, for simplicity, we will denote $u_{t}=u^{\prime}$. So, multiplying the first 
equation of (1.1) by $\bar{\psi}$, the second equation by $\phi^{\prime}$, integrating over $\Omega$ and making use of Green formula, we deduce that

$$
\begin{aligned}
& \int_{\Omega} \psi^{\prime} \bar{\psi} d x+i \int_{\Omega}|\nabla \psi|^{2} d x+\alpha \int_{\Omega}|\psi|^{4} d x=-i \int_{\Omega} \phi|\psi|^{2} d x \\
& \frac{1}{2} \frac{d}{d t} \int_{\Omega}\left(\left|\phi^{\prime}\right|^{2}+|\nabla \phi|^{2}\right) d x+\int_{\Omega} a(x)\left|\phi^{\prime}\right|^{2} d x=\int_{\omega}|\psi|^{2} \phi^{\prime} d x .
\end{aligned}
$$
obtain

Taking the real part in (3.1) and adding the obtained result with (3.2) we

$$
E^{\prime}(t)+\alpha \int_{\Omega}|\psi|^{4} d x+\int_{\Omega} a(x)\left|\phi^{\prime}\right|^{2} d x=\int_{\omega}|\psi|^{2} \phi^{\prime} d x .
$$

Next, we will analyze the last term on the RHS of (3.3). We have, from (2.3) and making use of the Cauchy-Schwarz inequality that

$$
\left.\left.\left|\int_{\omega}\right| \psi\right|^{2} \phi^{\prime} d x\left|\leq \frac{a_{0}^{-1}}{2} \int_{\omega}\right| \psi\right|^{4} d x+\frac{1}{2} \int_{\Omega} a(x)\left|\phi^{\prime}\right|^{2} d x .
$$

Combining (3.3) and (3.4) and considering $\alpha$ large enough such that $\beta:=$ $\alpha-\frac{a_{0}^{-1}}{2}>0$ it holds that

$$
E^{\prime}(t) \leq-\frac{1}{2} \int_{\Omega} a(x)\left|\phi^{\prime}\right|^{2} d x-\beta \int_{\Omega}|\psi|^{4} d x .
$$

In order to prove Theorem 2.1 it is sufficient to prove an estimate of type

$$
\int_{S}^{T} E^{2}(t) d t \leq C E(S), \quad \text { for all } 0 \leq S<T<+\infty
$$

for some positive constant $C$ that does not depend on $T$. Then, employing Theorem 9.1 of Kormonik's book $[\mathrm{K}]$ we deduce the desired decay rate in (2.8).

In order to prove inequality (3.6) we proceed in several steps.

Step 1. Multiplying the first equation of problem (1.1) by $E \bar{\psi}$ and the second equation by $E(t)(q \cdot \nabla \phi)$, where $q \in\left(W^{1, \infty}(\Omega)\right)^{n}$, and following (verbatim) the integration by parts of Lemma 3.7, Chap. I, of Lions [Li2] we deduce the following identity:

$$
\begin{aligned}
& {\left[E(t) \int_{\Omega}\left(\frac{|\psi|^{2}}{2}+\phi^{\prime}(q \cdot \nabla \phi)\right) d x\right]_{S}^{T}+\frac{1}{2} \int_{S}^{T} E(t) \int_{\Omega}(\operatorname{div} q)\left[\left|\phi^{\prime}\right|^{2}-|\nabla \phi|^{2}\right] d x d t} \\
& \quad+\int_{S}^{T} E(t) \int_{\Omega} \frac{\partial \phi}{\partial x_{j}} \frac{\partial q_{k}}{\partial x_{j}} \frac{\partial \phi}{\partial x_{k}} d x d t-\int_{S}^{T} E(t) \int_{\omega}|\psi|^{2}(q \cdot \nabla \phi) d x d t
\end{aligned}
$$




$$
\begin{aligned}
& +\int_{S}^{T} E(t) \int_{\Omega} a(x) \phi^{\prime}(q \cdot \nabla \phi) d x d t-\frac{1}{2} \int_{S}^{T} E^{\prime}(t) \int_{\Omega}|\psi|^{2} d x d t \\
& +\alpha \int_{S}^{T} E(t) \int_{\Omega}|\psi|^{4} d x d t-\int_{S}^{T} \int_{\Omega} E^{\prime}(q \cdot \nabla \phi) \phi^{\prime} d x d t \\
& =\frac{1}{2} \int_{S}^{T} E(t) \int_{\Gamma}(q \cdot \nu)\left(\frac{\partial \phi}{\partial \nu}\right)^{2} d \Gamma d t .
\end{aligned}
$$

In (3.7), for simplicity, we have omitted the variables of the functions under the integral signs and, in addition, we have used the convention of summation of repeated indexes.

Employing (3.7) with $q(x)=m(x)=x-x^{0}$, for some $x^{0} \in \mathbf{R}^{\mathbf{n}}$, and taking (2.5) into account, we arrive at

$$
\begin{aligned}
& {\left[E(t) \int_{\Omega}\left(\frac{|\psi|^{2}}{2}+\phi^{\prime}(m \cdot \nabla \phi)\right) d x\right]_{S}^{T}+\frac{n}{2} \int_{S}^{T} E(t) \int_{\Omega}\left[\left|\phi^{\prime}\right|^{2}-|\nabla \phi|^{2}\right] d x d t} \\
& \quad+\int_{S}^{T} E(t) \int_{\Omega}|\nabla \phi|^{2} d x d t-\int_{S}^{T} E(t) \int_{\omega}|\psi|^{2}(m \cdot \nabla \phi) d x d t \\
& \quad+\int_{S}^{T} E(t) \int_{\Omega} a(x) \phi^{\prime}(m \cdot \nabla \phi) d x d t-\frac{1}{2} \int_{S}^{T} E^{\prime}(t) \int_{\Omega}|\psi|^{2} d x d t \\
& \quad+\alpha \int_{S}^{T} E(t) \int_{\Omega}|\psi|^{4} d x d t-\int_{S}^{T} \int_{\Omega} E^{\prime}(m \cdot \nabla \phi) \phi^{\prime} d x d t \\
& \leq \\
& \frac{1}{2} \int_{S}^{T} E(t) \int_{\Gamma\left(x^{0}\right)}(m \cdot \nu)\left(\frac{\partial \phi}{\partial \nu}\right)^{2} d \Gamma d t .
\end{aligned}
$$

Now, multiplying the second equation of problem (1.1) by $E \xi \phi$, with $\xi \in$ $W^{1, \infty}(\Omega)$ and integrating by parts we obtain the following identity:

$$
\begin{aligned}
{[E(t)} & \left.\int_{\Omega} \phi \xi\left(\phi^{\prime}+\frac{\phi a}{2}\right) d x\right]_{S}^{T} \\
= & \int_{S}^{T} E(t) \int_{\omega}|\psi|^{2} \xi \phi d x d t+\int_{S}^{T} E(t) \int_{\Omega} \xi\left[\left|\phi^{\prime}\right|^{2}-|\nabla \phi|^{2}\right] d x d t-\int_{S}^{T} E(t) \int_{\Omega} \phi(\nabla \phi \\
& \cdot \nabla \xi) d x d t+\int_{S}^{T} E^{\prime} \int_{\Omega} \phi^{\prime} \xi \phi d x d t+\frac{1}{2} \int_{S}^{T} E^{\prime} \int_{\Omega} a \xi|\phi|^{2} d x d t
\end{aligned}
$$

Taking $\xi=\delta \in \mathbf{R}$ in (3.9) and combining the obtained result with (3.8) we have

$$
\begin{aligned}
& {\left[E(t) \int_{\Omega}\left(\frac{|\psi|^{2}}{2}+\phi^{\prime}(m \cdot \nabla \phi)+\delta \phi\left(\phi^{\prime}+\frac{\phi a}{2}\right)\right) d x\right]_{S}^{T}} \\
& \left.\quad+\left(\frac{n}{2}-\delta\right) \int_{S}^{T} E(t) \int_{\Omega}\left|\phi^{\prime}\right|^{2} d x d t+\left(1+\delta-\frac{n}{2}\right) \int_{S}^{T} E(t) \int_{\Omega}|\nabla \phi|^{2}\right] d x d t
\end{aligned}
$$




$$
\begin{aligned}
& -\int_{S}^{T} E(t) \int_{\omega}|\psi|^{2}(m \cdot \nabla \phi) d x d t+\int_{S}^{T} E(t) \int_{\Omega} a(x) \phi^{\prime}(m \cdot \nabla \phi) d x d t \\
& -\frac{1}{2} \int_{S}^{T} E^{\prime}(t) \int_{\Omega}|\psi|^{2} d x d t+\alpha \int_{S}^{T} E(t) \int_{\Omega}|\psi|^{4} d x d t \\
& -\delta \int_{S}^{T} E(t) \int_{\omega}|\psi|^{2} \phi d x d t-\delta \int_{S}^{T} E^{\prime}(t) \int_{\Omega} \phi^{\prime} \phi d x d t \\
& -\frac{\delta}{2} \int_{S}^{T} E^{\prime}(t) \int_{\Omega} a(x)|\phi|^{2} d x d t-\int_{S}^{T} \int_{\Omega} E^{\prime}(m \cdot \nabla \phi) \phi^{\prime} d x d t \\
& \leq \frac{1}{2} \int_{S}^{T} E(t) \int_{\Gamma\left(x^{0}\right)}(m \cdot \nu)\left(\frac{\partial \phi}{\partial \nu}\right)^{2} d \Gamma d t .
\end{aligned}
$$

Denoting

$$
\chi:=\left[E(t) \int_{\Omega}\left(\frac{|\psi|^{2}}{2}+\phi^{\prime}(m \cdot \nabla \phi)+\delta \phi\left(\phi^{\prime}+\frac{\phi a}{2}\right)\right) d x\right]_{S}^{T}
$$

and choosing $\delta=\frac{n-1}{2}$ if $n \geq 2$ (or $\delta \in(0,1 / 2)$ if $n=1$ ) we deduce

$$
\begin{aligned}
& \int_{S}^{T} E^{2}(t) d t+\chi-\frac{1}{2} \int_{S}^{T} E(t) \int_{\Omega}|\psi|^{2} d x d t \\
& \quad-\int_{S}^{T} E(t) \int_{\omega}|\psi|^{2}(m \cdot \nabla \phi) d x d t \\
& \quad+\int_{S}^{T} E(t) \int_{\Omega} a(x) \phi^{\prime}(m \cdot \nabla \phi) d x d t-\frac{1}{2} \int_{S}^{T} E^{\prime}(t) \int_{\Omega}|\psi|^{2} d x d t \\
& \quad+\alpha \int_{S}^{T} E(t) \int_{\Omega}|\psi|^{4} d x d t-\frac{n-1}{2} \int_{S}^{T} E(t) \int_{\omega}|\psi|^{2} \phi d x d t \\
& -\frac{n-1}{2} \int_{S}^{T} E^{\prime}(t) \int_{\Omega} \phi^{\prime} \phi d x d t-\frac{n-1}{4} \int_{S}^{T} E^{\prime}(t) \int_{\Omega} a(x)|\phi|^{2} d x d t \\
& -\int_{S}^{T} \int_{\Omega} E^{\prime}(m \cdot \nabla \phi) \phi^{\prime} d x d t \leq \frac{1}{2} \int_{S}^{T} E(t) \int_{\Gamma\left(x^{0}\right)}(m \cdot \nu)\left(\frac{\partial \phi}{\partial \nu}\right)^{2} d \Gamma d t .
\end{aligned}
$$

Next, we are going to estimate some terms in (3.12).

Estimate for $I_{1}:=-\frac{1}{2} \int_{S}^{T} E(t) \int_{\Omega}|\psi|^{2} d x d t$.

Making use of the inequality $a b \leq \frac{1}{4 \varepsilon} a^{2}+\varepsilon b^{2}$ and taking (2.2) and (3.5) into account, we infer

$$
\begin{aligned}
\left|I_{1}\right| & \leq \frac{k^{4}}{16 \varepsilon} \int_{S}^{T} \int_{\Omega}|\psi|^{4} d x d t+\varepsilon \int_{S}^{T} E^{2}(t) d t \\
& \leq-\frac{k^{4}}{16 \beta \varepsilon} \int_{S}^{T} E^{\prime}(t) d t+\varepsilon \int_{S}^{T} E^{2}(t) d t \\
& \leq \frac{k^{4}}{16 \beta \varepsilon} E(S)+\varepsilon \int_{S}^{T} E^{2}(t) d t .
\end{aligned}
$$


Estimate for $I_{2}:=-\int_{S}^{T} E(t) \int_{\omega}|\psi|^{2}(m \cdot \nabla \phi) d x d t$.

From now on we will denote

$$
R:=\sup _{x \in \bar{\Omega}} m(x)=\sup _{x \in \bar{\Omega}}\left|x-x^{0}\right| .
$$

So, making use of the integral Cauchy-Schwarz inequality, the numerical Hölder inequality, taking (2.7), (3.5) and (3.14) into account and also considering the inequality $a b \leq \frac{1}{4 \varepsilon} a^{2}+\varepsilon b^{2}$ we arrive at

$$
\begin{aligned}
\left|I_{2}\right| & \leq \frac{R^{2}}{4 \varepsilon} \int_{S}^{T} E(t) \int_{\Omega}|\psi|^{4} d x d t+2 \varepsilon \int_{S}^{T} E^{2}(t) d t \\
& \leq \frac{R^{2}}{8 \varepsilon \beta} E(0) E(S)+2 \varepsilon \int_{S}^{T} E^{2}(t) d t .
\end{aligned}
$$

Estimate for $I_{3}:=-\frac{n-1}{2} \int_{S}^{T} E(t) \int_{\omega}|\psi|^{2} \phi d x d t$.

Using Cauchy-Schwarz inequality, making use the inequality $a b \leq \frac{1}{4 \varepsilon} a^{2}$ $+\varepsilon b^{2}$ and taking (2.1), (2.7) and (3.5) into consideration, we can write

$$
\left|I_{3}\right| \leq \frac{(n-1)^{2} \lambda^{2}}{16 \varepsilon \beta} E(0) E(S)+2 \varepsilon \int_{S}^{T} E^{2}(t) d t
$$

Estimate for $I_{4}:=-\frac{1}{2} \int_{S}^{T} E^{\prime}(t) \int_{\Omega}|\psi|^{2} d x d t$

From (2.7) and (3.5) we deduce

$$
\left|I_{4}\right| \leq \frac{1}{2} \int_{S}^{T}\left|E^{\prime}(t)\right| E(t) d t=-\frac{1}{2} \int_{S}^{T}\left(E^{2}(t)\right)^{\prime} d t \leq \frac{1}{2} E(0) E(S) .
$$

Estimate for $I_{5}:=\alpha \int_{S}^{T} E(t) \int_{\Omega}|\psi|^{4} d x d t$.

Analogously, from (2.7) and (3.5) we obtain

$$
\left|I_{5}\right| \leq-\frac{\alpha}{\beta} \int_{S}^{T} E(t) E^{\prime}(t) d t \leq \frac{\alpha}{2 \beta} E(0) E(S) .
$$

Estimate for $I_{6}:=-\frac{n-1}{2} \int_{S}^{T} E^{\prime}(t) \int_{\Omega} \phi^{\prime} \phi d x d t$.

Making use of Cauchy-Schwarz inequality and taking (2.1), (2.7) and (3.5) into account, it holds that

$$
\begin{aligned}
\left|I_{6}\right| \leq \frac{(n-1) \lambda}{2} \int_{S}^{T}\left|E^{\prime}(t)\right| E(t) d t & \leq-\frac{(n-1) \lambda}{4} \int_{S}^{T}\left(E^{2}(t)\right)^{\prime} d t \\
& \leq \frac{(n-1) \lambda}{4} E(0) E(S) .
\end{aligned}
$$


Estimate for $I_{7}:=-\frac{n-1}{4} \int_{S}^{T} E^{\prime}(t) \int_{\Omega} a(x)|\phi|^{2} d x d t$.

From (2.1), (2.7) and (3.5) we deduce

$$
\begin{aligned}
\left|I_{7}\right| & \leq \frac{(n-1) \lambda^{2}}{4}\|a\|_{\infty} \int_{S}^{T}\left|E^{\prime}\right| \int_{\Omega}|\nabla \phi|^{2} d x d t \\
& \leq-\frac{(n-1) \lambda^{2}}{4}\|a\|_{\infty} \int_{S}^{T}\left(E^{2}(t)\right)^{\prime} d t \\
& \leq \frac{(n-1) \lambda^{2}}{4}\|a\|_{\infty} E(0) E(S) .
\end{aligned}
$$

Estimate for $I_{8}:=\int_{S}^{T} E(t) \int_{\Omega} a(x) \phi^{\prime}(m \cdot \nabla \phi) d x d t$.

Making use of the Cauchy-Schwarz inequality, employing the inequality $a b$ $\leq \frac{1}{4 \varepsilon} a^{2}+\varepsilon b^{2}$, and considering (2.7), (3.5) and (3.14) we obtain

$$
\left|I_{8}\right| \leq \frac{\|a\|_{\infty} R^{2}}{4 \varepsilon} E(0) E(S)+2 \varepsilon \int_{S}^{T} E^{2}(t) d t .
$$

Estimate for $I_{9}:=-\int_{S}^{T} \int_{\Omega} E^{\prime}(m \cdot \nabla \phi) \phi^{\prime} d x d t$.

From Cauchy-Schwarz inequality it holds that

$$
\left|I_{9}\right| \leq R \int_{S}^{T}\left|E^{\prime}(t)\right| E(t) d t \leq \frac{R}{2} E(0) E(S) .
$$

Combining (3.12)-(3.22) and choosing $\varepsilon=1 / 8$, the following inequality holds

$$
\begin{aligned}
\frac{1}{8} \int_{S}^{T} E^{2}(t) d t & \leq \frac{1}{2} \int_{S}^{T} E(t) \int_{\Gamma\left(x^{0}\right)}(m \cdot \nu)\left(\frac{\partial \phi}{\partial \nu}\right)^{2} d \Gamma d t \\
& +|\chi|+C_{0} E(S),
\end{aligned}
$$

where

$$
\begin{aligned}
C_{0}= & {\left[\frac{k^{4}+\left(2 R^{2}+2(n-1)^{2} \lambda^{2}+\alpha\right) E(0)}{2 \beta}\right.} \\
& \left.+\frac{\left(2+(n-1) \lambda+(n-1) \lambda^{2}\|a\|_{\infty}+8\|a\|_{\infty} R^{2}+2 R\right) E(0)}{4}\right] .
\end{aligned}
$$

Step 2. We now estimate the quantity $\frac{1}{2} \int_{S}^{T} E(t) \int_{\Gamma\left(x^{0}\right)}(m \cdot \nu)\left(\frac{\partial \phi}{\partial \nu}\right)^{2} d \Gamma d t$ in terms of the damping term $\int_{S}^{T} E(t) \int_{\Omega} a(x)\left|\phi^{\prime}\right|^{2} d x d t$.

According to the proof of Lemma 2.3 in Lions [Li2] we can construct a neighbourhood $\hat{\omega}$ of $\overline{\Gamma\left(x^{0}\right)}$ such that

$$
\overline{\hat{\omega}} \cap \Omega \subset \omega
$$


and a vector field $h \in\left(C^{1}(\bar{\Omega})\right)^{n}$ such that

$$
\begin{gathered}
h=\nu \text { on } \Gamma\left(x^{0}\right) ; \quad h \cdot \nu \geq 0 \text { a. e. in } \Gamma \text {, and } \\
h=0 \text { on } \Omega \backslash \hat{\omega},
\end{gathered}
$$

according to the figure 2 below.

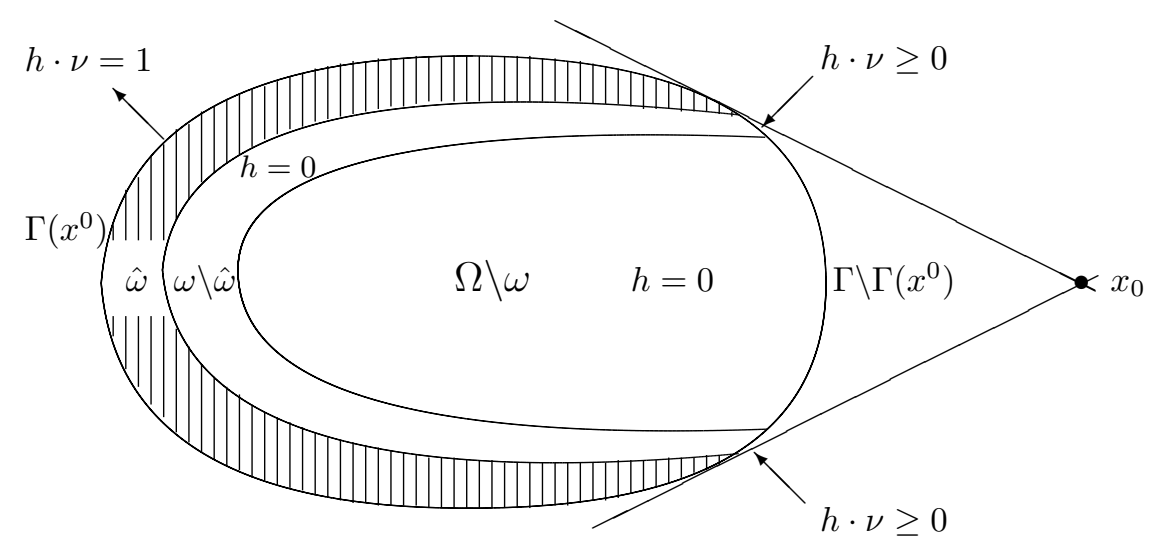

Figure 2

Applying the identity in (3.7) with $q=h$ it holds that

$$
\begin{aligned}
& \frac{1}{2} \int_{S}^{T} E(t) \int_{\Gamma\left(x^{0}\right)}\left(\frac{\partial \phi}{\partial \nu}\right)^{2} d \Gamma d t \\
& \leq \frac{1}{2} \int_{S}^{T} E(t) \int_{\Gamma\left(x^{0}\right)} \underbrace{(h \cdot \nu)}_{=1}\left(\frac{\partial \phi}{\partial \nu}\right)^{2} d \Gamma d t \\
& +\frac{1}{2} \int_{S}^{T} E(t) \int_{\Gamma \backslash \Gamma\left(x^{0}\right)} \underbrace{(h \cdot \nu)}_{\geq 0}\left(\frac{\partial \phi}{\partial \nu}\right)^{2} d \Gamma d t \\
& =\left[E(t) \int_{\Omega}\left(\frac{|\psi|^{2}}{2}+\phi^{\prime}(h \cdot \nabla \phi)\right) d x\right]_{S}^{T}+\frac{1}{2} \int_{S}^{T} E(t) \int_{\hat{\omega}}(\operatorname{div} h)\left[\left|\phi^{\prime}\right|^{2}-|\nabla \phi|^{2}\right] d x d t \\
& +\int_{S}^{T} E(t) \int_{\hat{\omega}} \frac{\partial \phi}{\partial x_{j}} \frac{\partial h_{k}}{\partial x_{j}} \frac{\partial \phi}{\partial x_{k}} d x d t-\int_{S}^{T} E(t) \int_{\hat{\omega}}|\psi|^{2}(h \cdot \nabla \phi) d x d t \\
& +\int_{S}^{T} E(t) \int_{\hat{\omega}} a(x) \phi^{\prime}(h \cdot \nabla \phi) d x d t-\frac{1}{2} \int_{S}^{T} E^{\prime}(t) \int_{\Omega}|\psi|^{2} d x d t \\
& +\alpha \int_{S}^{T} E(t) \int_{\Omega}|\psi|^{4} d x d t-\int_{S}^{T} \int_{\Omega} E^{\prime}(h \cdot \nabla \phi) \phi^{\prime} d x d t .
\end{aligned}
$$

In what follows we will estimate some terms on the RHS of (3.27). 
Estimate for $J_{1}:=-\frac{1}{2} \int_{S}^{T} E^{\prime}(t) \int_{\Omega}|\psi|^{2} d x d t$.

From (2.7) and (3.5) we deduce

$$
\left|J_{1}\right| \leq-\int_{S}^{T} E^{\prime}(t) E(t) d t \leq \frac{1}{2} E(0) E(S) .
$$

Estimate for $J_{2}:=\alpha \int_{S}^{T} E(t) \int_{\Omega}|\psi|^{4} d x d t$.

Analogously, from (2.7) and (3.5) we have

$$
\left|J_{2}\right| \leq-\frac{\alpha}{\beta} \int_{S}^{T} E(t) E^{\prime}(t) d t \leq \frac{\alpha}{2 \beta} E(0) E(S) .
$$

Estimate for $J_{3}:=-\int_{S}^{T} E(t) \int_{\hat{\omega}}|\psi|^{2}(h \cdot \nabla \phi) d x d t$.

Using Cauchy-Schwarz and Hölder inequalities, making use the inequality $a b \leq \frac{1}{4 \varepsilon} a^{2}+\varepsilon b^{2}$ and taking (2.7) and (3.5) into consideration, we can write

$$
\begin{aligned}
\left|J_{3}\right| & \leq \frac{\|h\|_{\infty}^{2}}{4 \varepsilon} \int_{S}^{T} E(t) \int_{\Omega}|\psi|^{4} d x d t+2 \varepsilon \int_{S}^{T} E^{2}(t) d t \\
& \leq-\frac{\|h\|_{\infty}^{2}}{4 \varepsilon \beta} \int_{S}^{T} E(t) E^{\prime}(t) d t+2 \varepsilon \int_{S}^{T} E^{2}(t) d t \\
& \leq \frac{\|h\|_{\infty}^{2}}{8 \varepsilon \beta} E(0) E(S)+2 \varepsilon \int_{S}^{T} E^{2}(t) d t .
\end{aligned}
$$

Estimate for $J_{4}:=\int_{S}^{T} E(t) \int_{\hat{\omega}} a(x) \phi^{\prime}(h \cdot \nabla \phi) d x d t$.

From (2.7), (3.5) and analogously we have done above we obtain

$$
\left|J_{4}\right| \leq \frac{\|h\|_{\infty}^{2}\|a\|_{\infty}}{8 \varepsilon} E(0) E(S)+2 \varepsilon \int_{S}^{T} E^{2}(t) d t .
$$

Estimate for $J_{5}:=\frac{1}{2} \int_{S}^{T} E(t) \int_{\hat{\omega}}(\operatorname{div} h)\left[\left|\phi^{\prime}\right|^{2}-|\nabla \phi|^{2}\right] d x d t$.

From (2.3), (2.7) and (3.5) we infer

$$
\left|J_{5}\right| \leq \frac{\|h\|_{W^{1, \infty}}}{2 a_{0}} E(0) E(s)+\frac{1}{2}|| h \|_{W^{1, \infty}} \int_{S}^{T} E(t) \int_{\hat{\omega}}|\nabla \phi|^{2} d x d t .
$$

Estimate for $J_{6}:=\int_{S}^{T} E(t) \int_{\hat{\omega}} \frac{\partial \phi}{\partial x_{j}} \frac{\partial h_{k}}{\partial x_{j}} \frac{\partial \phi}{\partial x_{k}} d x d t$.

We have

$$
\left|J_{6}\right| \leq\|h\|_{W^{1, \infty}} \int_{S}^{T} E(t) \int_{\hat{\omega}}|\nabla \phi|^{2} d x d t
$$


Estimate for $J_{7}:=-\int_{S}^{T} \int_{\Omega} E^{\prime}(h \cdot \nabla \phi) \phi^{\prime} d x d t$. We have,

$$
\left|J_{7}\right| \leq\|h\|_{\infty} \int_{S}^{T}\left|E^{\prime}(t)\right| E(t) d t \leq \frac{\|h\|_{\infty}}{2} E(0) E(S)
$$

Denoting

$$
Y:=\left[E(t) \int_{\Omega}\left(\frac{|\psi|^{2}}{2}+\phi^{\prime}(h \cdot \nabla \phi)\right) d x\right]_{S}^{T}
$$

and combining (3.27)-(3.35), we obtain

$$
\begin{aligned}
\frac{R}{2} \int_{S}^{T} E(t) \int_{\Gamma\left(x^{0}\right)}\left(\frac{\partial \phi}{\partial \nu}\right)^{2} d \Gamma d t & \leq R|Y|+4 R \varepsilon \int_{S}^{T} E^{2}(t) d t+R C_{1} E(S)(3 . \\
& +\frac{3 R}{2}\|h\|_{W^{1, \infty}} \int_{S}^{T} E(t) \int_{\hat{\omega}}|\nabla \phi|^{2} d x d t
\end{aligned}
$$

where

$$
C_{1}:=\left[\frac{1}{2}+\frac{\alpha}{2 \beta}+\frac{\|h\|_{\infty}^{2}}{8 \varepsilon \beta}+\frac{\|a\|_{\infty}\|h\|_{\infty}^{2}}{8 \varepsilon}+\frac{\|h\|_{W^{1, \infty}}}{2 a_{0}}+\frac{\|h\|_{\infty}}{2}\right] E(0) .
$$

Combining (3.23) and (3.36) and choosing $\varepsilon=1 / 64 R$ we deduce

$$
\begin{aligned}
& \frac{1}{16} \int_{S}^{T} E^{2}(t) d t \\
& \leq|\chi|+R|Y|+\left(C_{0}+R C_{1}\right) E(S) \frac{3 R}{2}\|h\|_{W^{1, \infty}} \int_{S}^{T} E(t) \int_{\hat{\omega}}|\nabla \phi|^{2} d x d t .
\end{aligned}
$$

Then, we construct, as in Lemma 2.4 in Lions $[\mathrm{Li}]$ a function $\eta \in W^{1, \infty}(\Omega)$ satisfying

$$
\begin{gathered}
0 \leq \eta \leq 1 \text { a.e. in } \Omega ; \quad \eta=1 \text { a. e. in } \hat{\omega}, \\
\eta=0 \text { a. e. in } \Omega \backslash \omega \\
\frac{|\nabla \eta|^{2}}{\eta} \in L^{\infty}(\omega) .
\end{gathered}
$$

Taking $\xi=\eta$ in the identity (3.9) it results that

$$
\begin{aligned}
& {\left[E(t) \int_{\omega} \phi \eta\left(\phi^{\prime}+\frac{\phi a}{2}\right) d x\right]_{S}^{T}=\int_{S}^{T} E(t) \int_{\omega}|\psi|^{2} \eta \phi d x d t} \\
& \quad+\int_{S}^{T} E(t) \int_{\omega} \eta\left[\left|\phi^{\prime}\right|^{2}-|\nabla \phi|^{2}\right] d x d t-\int_{S}^{T} E(t) \int_{\omega} \phi(\nabla \phi \cdot \nabla \eta) d x d t \\
& \quad+\int_{S}^{T} E^{\prime}(t) \int_{\omega} \phi^{\prime} \eta \phi d x d t+\frac{1}{2} \int_{S}^{T} E^{\prime}(t) \int_{\omega} a(x) \eta|\phi|^{2} d x d t
\end{aligned}
$$


Next, let us analyze the terms on the RHS of (3.41).

Estimate for $L_{1}:=\int_{S}^{T} E^{\prime}(t) \int_{\omega} \phi^{\prime} \eta \phi d x d t$.

From (2.1), (2.7) and taking (3.5) and (3.38) into account, it holds that

$$
\left|L_{1}\right| \leq \frac{1}{2} \lambda E(0) E(S)
$$

Estimate for $L_{2}:=\frac{1}{2} \int_{S}^{T} E^{\prime}(t) \int_{\omega} a(x) \eta|\phi|^{2} d x$.

Analogously, we deduce that

$$
\left|L_{2}\right| \leq \frac{\lambda^{2}\|a\|_{\infty}}{2} E(0) E(S) .
$$

Estimate for $L_{3}:=\int_{S}^{T} E(t) \int_{\omega}|\psi|^{2} \eta \phi d x d t$.

Analogously to the above estimates and now considering the inequality $a b \leq \frac{1}{4 \varepsilon} a^{2}+\varepsilon b^{2}$, it follows that

$$
\left|L_{3}\right| \leq \frac{1}{8 \varepsilon \beta} E(0) E(S)+2 \varepsilon \lambda^{2} \int_{S}^{T} E^{2}(t) d t .
$$

Estimate for $L_{4}:=\int_{S}^{T} E(t) \int_{\omega} \eta\left|\phi^{\prime}\right|^{2} d x d t$.

From (2.3), (2.7), (3.5) and (3.38) we arrive at

$$
\left|L_{4}\right| \leq a_{0}^{-1} E(0) E(S) .
$$

Estimate for $L_{5}:=-\int_{S}^{T} E(t) \int_{\omega} \phi(\nabla \phi \cdot \nabla \eta) d x d t$.

From (3.38)-(3.40), we can write

$$
\begin{aligned}
\left|L_{5}\right| \leq & \frac{1}{2} \int_{S}^{T} E(t) \int_{\omega} \eta|\nabla \phi|^{2} d x \\
& +\frac{1}{2}\left\|\frac{|\nabla \eta|^{2}}{\eta}\right\|_{L^{\infty}(\omega)} \int_{S}^{T} E(t) \int_{\omega}|\phi|^{2} d x d t .
\end{aligned}
$$

Defining

$$
Z:=\left[E(t) \int_{\omega} \phi \eta\left(\phi^{\prime}+\frac{\phi a}{2}\right) d x\right]_{S}^{T}
$$

and combining (3.41)-(3.47) we obtain

$$
\begin{aligned}
& \int_{S}^{T} E(t) \int_{\omega} \eta|\nabla \phi|^{2} d x d t \leq|Z|+C_{2} E(S)+2 \varepsilon \lambda^{2} \int_{S}^{T} E^{2}(t) d t \\
& +\frac{1}{2} \int_{S}^{T} E(t) \int_{\omega} \eta|\nabla \phi|^{2} d x+\frac{1}{2}\left\|\frac{|\nabla \eta|^{2}}{\eta}\right\|_{L^{\infty}(\omega)} \int_{S}^{T} E(t) \int_{\omega}|\phi|^{2} d x d t
\end{aligned}
$$


where

$$
C_{2}:=\left[\frac{\lambda}{2}+\frac{\lambda^{2}\|a\|_{\infty}}{2}+\frac{1}{8 \varepsilon \beta}+a_{0}^{-1}\right] E(0) .
$$

Combining (3.37) and (3.48), choosing $\varepsilon=1 / 64 \lambda^{2}$ and having in mind that

$$
\int_{S}^{T} E(t) \int_{\hat{\omega}} \eta|\nabla \phi|^{2} d x d t=\int_{S}^{T} E(t) \int_{\hat{\omega}}|\nabla \phi|^{2} d x d t
$$

we deduce

$$
\begin{aligned}
& \frac{1}{32} \int_{S}^{T} E^{2}(t) d t \\
& \leq|\chi|+R|Y|+|Z|+\left(C_{0}+R C_{1}+3 C_{2}\|h\|_{\infty}\right) E(S) \\
& \quad+\frac{3 R}{4}|| h\left\|_{W^{1, \infty}}\right\| \frac{|\nabla \eta|^{2}}{\eta} \|_{L^{\infty}(\omega)} \int_{S}^{T} E(t) \int_{\omega}|\phi|^{2} d x d t .
\end{aligned}
$$
holds

On the other hand, from (3.11), (3.35) and (3.47), the following estimate

$$
|\chi|+R|Y|+|Z| \leq C_{3} E(0) E(S)
$$

where $C_{3}$ is a positive constant such that $C_{3}=C_{3}\left(R,\|a\|_{\infty}, \lambda,\|h\|_{\infty}\right)$. Then, (3.49) and (3.50) yield

$$
\int_{S}^{T} E^{2}(t) d t \leq C E(0) E(S)+C \int_{S}^{T} E(t) \int_{\Omega}|\phi|^{2} d x d t,
$$

where $C$ is a positive constant such that

$$
C=C\left(R,\|a\|_{\infty},\|h\|_{\infty}, \lambda,\|h\|_{W^{1, \infty}}, k, n, \alpha, \beta, a_{0}\right) .
$$

Step 3. Let $T_{0}>0$ considered sufficiently large for our purpose. We will prove the following lemma:

Lemma 3.1 For all $T>T_{0}$ there exists a positive constant $C=C\left(T_{0}, E(0)\right)$ such that if $(\psi, \phi)$ is the regular solution of (1.1) with initial data $\left\{\psi_{0}, \phi_{0}, \phi_{1}\right\}$ we have

$$
\begin{aligned}
& \int_{S}^{T} \int_{\Omega}|\phi|^{2} d x d t \\
& \quad \leq C\left(T_{0}, E(0)\right)\left[\int_{S}^{T} \int_{\Omega} a(x)\left|\phi^{\prime}\right|^{2} d x d t+\int_{S}^{T} \int_{\Omega}|\psi|^{4} d x d t\right]
\end{aligned}
$$

for $0 \leq S<T<+\infty$. 
Proof. We argue by contradiction. Let us suppose that (3.52) is not verified and let $\left\{\psi_{k}(0), \phi_{k}(0), \phi_{k}^{\prime}(0)\right\}$ be a sequence of initial data where the corresponding solutions $\left\{\psi_{k}, \phi_{k}\right\}$ with $E_{k}(0)$ uniformly bounded in $k$, verifies

$$
\lim _{k \rightarrow+\infty} \frac{\int_{S}^{T} \int_{\Omega}\left|\phi_{k}\right|^{2} d x d t}{\int_{S}^{T} \int_{\Omega} a(x)\left|\phi_{k}^{\prime}\right|^{2} d x d t+\int_{S}^{T} \int_{\Omega}\left|\psi_{k}\right|^{4} d x d t}=+\infty .
$$

Since $E_{k}(t)$ is non-increasing and $E_{k}(0)$ remains bounded then, we obtain a subsequence, still denoted by $\left\{\psi_{k}, \phi_{k}\right\}$ which verifies

$$
\begin{aligned}
& \psi_{k} \rightarrow \psi \text { weak star in } L^{\infty}\left(0, T ; L^{2}(\Omega)\right), \\
& \phi_{k} \rightarrow \phi \text { weak star in } L^{\infty}\left(0, T ; H_{0}^{1}(\Omega)\right), \\
& \phi_{k}^{\prime} \rightarrow \phi^{\prime} \text { weak star in } L^{\infty}\left(0, T ; L^{2}(\Omega)\right), \\
& \psi_{k} \rightarrow \psi \text { weakly in } L^{4}\left(0, T ; L^{4}(\Omega)\right),
\end{aligned}
$$

We also have, employing compactness results (see Theorem 5.1 in Lions [Li]) that

$$
\phi_{k} \rightarrow \phi \text { strongly in } L^{2}\left(0, T ; L^{2}(\Omega)\right) .
$$

Now, from (3.53) and (3.55) we deduce that

$$
\begin{gathered}
\lim _{k \rightarrow+\infty} \int_{S}^{T} \int_{\Omega} a(x)\left|\phi_{k}^{\prime}\right|^{2} d x d t=0, \\
\lim _{k \rightarrow+\infty} \int_{S}^{T} \int_{\Omega}\left|\psi_{k}\right|^{4} d x d t=0,
\end{gathered}
$$

From now on let us focus our attention on the coupled wave equation

$$
\phi_{k}^{\prime \prime}-\Delta \phi_{k}+a(x) \phi_{k}^{\prime}=\left|\psi_{k}\right|^{2} \chi_{\omega} \text { in } \Omega \times(0, T)
$$

Let us divide our proof in two cases (in what concerns the limit $\phi$ above):

(a) $\phi \neq 0$

Passing to the limit when $k \rightarrow+\infty$ in (3.61) taking into account the above convergence, we deduce that

$$
\left\{\begin{array}{l}
\phi^{\prime \prime}-\Delta \phi=0 \text { in } L^{2}\left(0, T ; H^{-1}(\Omega)\right) \\
\phi=0 \text { on } L^{2}\left(0, T ; H^{1 / 2}(\Gamma)\right) \\
\phi^{\prime}=0 \text { a. e. in } \omega \times(0, T)
\end{array}\right.
$$

and for $\phi^{\prime}=v$, we obtain, in the distributional sense that

$$
\left\{\begin{array}{l}
v^{\prime \prime}-\Delta v=0 \text { in } D^{\prime}(\Omega \times(0, T)) \\
v=0 \text { on } \Gamma \times(0, T)\left(\text { in } H^{-1}\left(0, T ; H^{1 / 2}(\Gamma)\right)\right) \\
v=0 \text { a. e. in } \omega \times(0, T) .
\end{array}\right.
$$


From standard uniqueness results for the wave equation we conclude that $v \equiv 0$, that is, $\phi^{\prime} \equiv 0$. Returning to (3.62) we obtain the following elliptic equation for a. e. $t \in(0, T)$ :

$$
\left\{\begin{array}{l}
-\Delta \phi=0 \text { in } \Omega \\
\phi=0 \text { on } \Gamma \\
\phi^{\prime}=0 \text { in } \omega,
\end{array}\right.
$$

Multiplying (3.64) by $\phi$ we deduce that $\int_{\Omega}|\nabla \phi|^{2} d x=0$, which implies that $\phi \equiv 0$, which is a contradiction.

Now, we consider the other case when

(b) $\phi \equiv 0$.

Defining

we obtain

$$
\begin{aligned}
c_{k} & :=\left[\int_{S}^{T} \int_{\Omega}\left|\phi_{k}\right|^{2} d x d t\right]^{1 / 2} \\
\hat{\phi}_{k} & =\frac{1}{c_{k}} \phi_{k}, \quad \hat{\psi}_{k}=\frac{1}{c_{k}} \psi_{k}
\end{aligned}
$$

$$
\int_{S}^{T} \int_{\Omega}\left|\hat{\phi}_{k}\right|^{2} d x d t=1
$$

Besides,

$$
\begin{aligned}
\hat{E}_{k}(t) & =\frac{1}{2}\left[\int_{\Omega}\left|\hat{\psi}_{k}\right|^{2} d x+\int_{\Omega}\left|\hat{\phi}_{k}^{\prime}\right|^{2} d x+\int_{\Omega}\left|\nabla \hat{\phi}_{k}\right|^{2} d x\right] \\
& =\frac{1}{2 c_{k}^{2}}\left[\int_{\Omega}\left|\psi_{k}\right|^{2} d x+\int_{\Omega}\left|\phi_{k}^{\prime}\right|^{2} d x+\int_{\Omega}\left|\nabla \phi_{k}\right|^{2} d x\right],
\end{aligned}
$$

that is,

$$
\hat{E}_{k}(t)=\frac{1}{2 c_{k}^{2}} E_{k}(t) .
$$

On the other hand, multiplying $(3.3)$ by $E_{k}(t)$ and integrating over $(S, T)$, we deduce

$$
\begin{aligned}
E_{k}^{2}(T)= & E_{k}^{2}(S)-2 \alpha \int_{S}^{T} E_{k}(t) \int_{\Omega}\left|\psi_{k}\right|^{4} d x d t-2 \int_{S}^{T} E_{k}(t) \int_{\Omega} a(x)\left|\phi_{k}^{\prime}\right|^{2} d x d t \\
& +2 \int_{S}^{T} E_{k}(t) \int_{\omega}\left|\psi_{k}\right|^{2} \phi_{k}^{\prime} d x d t .
\end{aligned}
$$

From the fact that $E_{k}(t) \geq E_{k}(T)$ for all $t \in[S, T]$ and taking (3.67) into account, we obtain

$$
\begin{aligned}
\int_{S}^{T} E_{k}^{2}(t) d t & \geq[T-S] E_{k}^{2}(T) \\
& =[T-S] E_{k}^{2}(S)-2 \alpha[T-S] \int_{S}^{T} E_{k}(t) \int_{\Omega}\left|\psi_{k}\right|^{4} d x d t
\end{aligned}
$$




$$
\begin{aligned}
& -2[T-S] \int_{S}^{T} E_{k}(t) \int_{\Omega} a(x)\left|\phi_{k}^{\prime}\right|^{2} d x d t \\
& +2[T-S] \int_{S}^{T} E_{k}(t) \int_{\omega}\left|\psi_{k}\right|^{2} \phi_{k}^{\prime} d x d t .
\end{aligned}
$$

Combining (3.51) and (3.70) and making use of Cauchy-Schwarz inequality taking (2.3) into account, we infer

$$
\begin{aligned}
& {[T-S] E_{k}(S) E_{k}(S)} \\
& \leq\left(2(\alpha+2)+2 a_{0}^{-1}\right)[T-S] E_{k}(S)\left\{\int_{S}^{T} \int_{\Omega}\left|\psi_{k}\right|^{4} d x d t+\int_{S}^{T} \int_{\Omega} a(x)\left|\phi_{k}^{\prime}\right|^{2} d x d t\right\} \\
& \quad+C E_{k}(S)+C E_{k}(S) \int_{S}^{T} \int_{\Omega}\left|\phi_{k}\right|^{2} d x d t .
\end{aligned}
$$

The last inequality yields for a large $T$,

$$
\begin{aligned}
E_{k}(S) \leq C\left(T, a_{0}, \alpha\right)\left\{\int_{S}^{T} \int_{\Omega}\left|\psi_{k}\right|^{4} d x d t\right. & \int_{S}^{T} \int_{\Omega} a(x)\left|\phi_{k}^{\prime}\right|^{2} d x d t \\
& \left.+\int_{S}^{T} \int_{\Omega}\left|\phi_{k}\right|^{2} d x d t\right\}
\end{aligned}
$$

Having in mind that $E_{k}(t) \leq E_{k}(S)$ for all $t \in[S, T]$, applying inequality (3.71) and dividing both sides by $\int_{S}^{T} \int_{\Omega}\left|\phi_{k}\right|^{2} d x d t$ it holds that

$$
\frac{E_{k}(t)}{\int_{S}^{T} \int_{\Omega}\left|\phi_{k}\right|^{2} d x d t} \leq C\left(T, a_{0}, \alpha\right)\left\{\frac{\int_{S}^{T} \int_{\Omega}\left|\psi_{k}\right|^{4} d x d t+\int_{S}^{T} \int_{\Omega} a(x)\left|\phi_{k}^{\prime}\right|^{2} d x d t}{\int_{S}^{T} \int_{\Omega}\left|\phi_{k}\right|^{2} d x d t}+1\right\}
$$

Since in view of (3.53) we have

$$
\lim _{k \rightarrow+\infty} \frac{\int_{S}^{T} \int_{\Omega}\left|\psi_{k}\right|^{4} d x d t+\int_{S}^{T} \int_{\Omega} a(x)\left|\phi_{k}^{\prime}\right|^{2} d x d t}{\int_{S}^{T} \int_{\Omega}\left|\phi_{k}\right|^{2} d x d t}=0,
$$

then, from (3.72) there exists $M>0$ such that

$$
\begin{array}{r}
\frac{E_{k}(t)}{c_{k}^{2}} \leq C\left(T, a_{0}, \alpha\right)(M+1), \text { for all } t \in[S, T] \\
\text { and for all } k \in \mathbf{N} .
\end{array}
$$

Consequently, from (3.68) and (3.74) it results that

$$
\begin{array}{r}
\hat{E}_{k}(t) \leq C\left(T, a_{0}, \alpha\right)(M+1), \text { for all } t \in[S, T] \\
\text { and for all } k \in \mathbf{N} .
\end{array}
$$


Then, in particular, from (3.73) we deduce

$$
\begin{gathered}
\lim _{k \rightarrow+\infty} \int_{S}^{T} \int_{\Omega} a(x)\left|\hat{\phi}_{k}^{\prime}\right|^{2} d x d t=\lim _{k \rightarrow+\infty} \frac{\int_{S}^{T} \int_{\Omega} a(x)\left|\phi_{k}^{\prime}\right|^{2} d x d t}{\int_{S}^{T} \int_{\Omega}\left|\phi_{k}\right|^{2} d x d t}=0, \\
\lim _{k \rightarrow+\infty} \frac{\int_{S}^{T} \int_{\Omega}\left|\psi_{k}\right|^{4} d x d t}{\int_{S}^{T} \int_{\Omega}\left|\phi_{k}\right|^{2} d x d t}=0
\end{gathered}
$$

and from and (3.75), for a subsequence $\left\{\psi_{k}, \phi_{k}\right\}$, we obtain

$$
\begin{aligned}
& \hat{\psi}_{k} \rightarrow \hat{\psi} \text { weak star in } L^{\infty}\left(0, T ; L^{2}(\Omega)\right), \\
& \hat{\phi}_{k} \rightarrow \hat{\phi} \text { weak star in } L^{\infty}\left(0, T ; H_{0}^{1}(\Omega)\right), \\
& \hat{\phi}_{k}^{\prime} \rightarrow \hat{\phi}^{\prime} \quad \text { weak star in } L^{\infty}\left(0, T ; H_{0}^{1}(\Omega)\right), \\
& \hat{\phi}_{k} \rightarrow \hat{\phi} \text { strongly in } L^{2}\left(0, T ; L^{2}(\Omega)\right) .
\end{aligned}
$$

In addition, $\hat{\phi}_{k}$ satisfies the equation

$$
\left\{\begin{array}{l}
\hat{\phi}_{k}^{\prime \prime}-\Delta \hat{\phi}_{k}+a(x) \hat{\phi}_{k}^{\prime}=\frac{\left|\psi_{k}\right|^{2}}{c_{k}} \text { in } \Omega \times(0, T) \\
\hat{\phi}_{k}=0 \text { on } \Gamma \times(0, T) \\
\hat{\phi}_{k}^{\prime}=0 \text { a. e. in } \omega \times(0, T) .
\end{array}\right.
$$

Passing to the limit when $k \rightarrow+\infty$ taking the above convergence into account, we get

$$
\left\{\begin{array}{l}
\hat{\phi}^{\prime \prime}-\Delta \hat{\phi}=0 \text { in } \Omega \times(0, T) \\
\hat{\phi}=0 \text { on } \Gamma \times(0, T) \\
\hat{\phi}^{\prime}=0 \text { a. e. in } \omega \times(0, T) .
\end{array}\right.
$$

Then, $v=\hat{\phi}^{\prime}$ verifies, in the distributional sense

$$
\left\{\begin{array}{l}
v^{\prime \prime}-\Delta v=0 \text { in } D^{\prime}(\Omega \times(0, T)) \\
v=0 \text { on } \Gamma \times(0, T)(\text { in a weak sense }) \\
v=0 \text { a. e. in } \omega \times(0, T) .
\end{array}\right.
$$

Applying uniqueness standard results it comes that $v=\hat{\phi}^{\prime}=0$. Returning to $(3.83)$ we obtain, for a. e. $t \in(0, T)$ that

$$
\left\{\begin{array}{l}
-\Delta \hat{\phi}=0 \text { in } \Omega \\
\hat{\phi}=0 \text { on } \Gamma \\
\hat{\phi}^{\prime}=0 \text { in } \omega .
\end{array}\right.
$$

Multiplying the above equation by $\hat{\phi}$, we deduce

$$
0=-\int_{\Omega} \Delta \hat{\phi} \hat{\phi} d x=\int_{\Omega}|\nabla \hat{\phi}|^{2} d x
$$


that is, $\hat{\phi}=0$. From this fact, from (3.67) and (3.81) we obtain a contradiction. So, Lemma 3.1 is proved.

Combining (3.51) and (3.52) it holds that

$$
\int_{S}^{T} E^{2}(t) d t \leq C E(0) E(S)
$$

where $C=C\left(T_{0}, E(0), R,\|a\|_{\infty},\|h\|_{\infty}, \lambda,\|h\|_{W^{1, \infty}}, k, n, \alpha, \beta, a_{0}\right)$, independent of $T$. So, the estimate given in (3.6) is proved as desired to show. Consequently Theorem 2.1 is proved.

Remark 3.1 Following the method developed in this paper combined with those ones introduced in the literature by Zuazua [Zua] it is also possible to treat semi-linear coupled waves, that is, systems given by

$$
\left\{\begin{array}{l}
i \psi_{t}+\Delta \psi+i \alpha|\psi|^{2} \psi=\phi \psi \text { in } \Omega \times(0, \infty) \\
\phi_{t t}-\Delta \phi+f(\phi)+a(x) \phi_{t}=|\psi|^{2} \chi_{\omega} \text { in } \Omega \times(0, \infty) \\
\psi(0)=\psi_{0} \in H_{0}^{1}(\Omega), u(0)=u_{0} \in H_{0}^{1}(\Omega), u_{t}(0)=u_{1} \in L^{2}(\Omega),
\end{array}\right.
$$

where $f$ is a real function satisfying the following assumptions:

$$
f \in C^{1}(R), f(0)=0, f(s) s>0 \text { for all } s \neq 0,
$$

and

$$
\begin{aligned}
& \left|f^{\prime}(s)\right| \leq C\left[1+|s|^{k_{0}-1}\right], \quad \text { for all } s \in \mathbb{R}, 1 \leq k_{0} \leq \frac{n}{n-2}, n>2 \\
& \text { and } 0<k_{0}<\infty, n=1,2 .
\end{aligned}
$$

Remark 3.2 As mentioned in the introduction of this paper, an interesting open question is to investigate uniform decay rates when one has localized dissipations in both equations, namely,

$$
\left\{\begin{array}{l}
i \psi_{t}+\Delta \psi+i \alpha b(x)|\psi|^{2} \psi=\phi \psi \text { in } \Omega \times(0, \infty) \\
\phi_{t t}-\Delta \phi+a(x) \phi_{t}=|\psi|^{2} \chi_{\omega} \text { in } \Omega \times(0, \infty) \\
\psi(0)=\psi_{0} \in H_{0}^{1}(\Omega), u(0)=u_{0} \in H_{0}^{1}(\Omega), u_{t}(0)=u_{1} \in L^{2}(\Omega)
\end{array}\right.
$$

where

$$
a(x) \geq a_{0}>0 \text { and } \quad b(x) \geq b_{0}>0 \quad \text { a. e. in } \omega .
$$

Unfortunately, the method developed in the present manuscript fails, mainly because of the coupled Schrödinger equation. Perhaps, it would be interesting to investigate the case where we have localized damping acting in the whole domain. More precisely, assume that $a, b \in L_{+}^{\infty}(\Omega)$ verifying

$$
a(x)+b(x) \geq \alpha_{0}>0 \quad \text { a. e. in } \Omega,
$$

as considered in Cavalcanti and Oquendo [CaOq] for the viscoelastic wave equation. 


\section{Acknowledgement}

The authors would like to thank the anonymous referee for his(her) useful remarks.

\section{References}

[Ba] A. BACHELOT, Problème de Cauchy pour des systèmes hyperboliques semi-linéares, Ann. Inst. H. Poincaré Anal. non Linéaire 1 (1984), 453-478.

[BaCha] J. B. BAILLON and J. M. CHADAM, The Cauchy problem for the coupled Klein-Gordon-Schrödinger equations, in Contemporary Developments in Continuum Mechanics and Partial Differential Equations, North Holland, Amsterdam, (1978), 37-44.

[Be-He-Lo] R. BEY, A. HEMINNA and J.-P. LOHAC, Boundary stabilization of the linear elastodynamic system by a Lyapunov type method, Rev. Mat. Complut. 16(2) (2003), 417-441.

[BoYo1] B. GUO and Y. Li, Attractor for dissipative Klein-Gordon-Schrödinder equations in $\mathbf{R}^{\mathbf{3}}$, Journal of Differential Equations 136 (1997), 356-377.

[BoYo2] B. GUO and Y. LI, Attractor for the dissipative generalized KleinGordon-Schrödinger equations, J. Partial Differ. Equations 11(3) (1998), 260-272.

[BoYo3] B. GUO and Y. LI, Asymptotic smoothing effect of solutions to weakly dissipative Klein-Gordon-Schrödinger equations, J. Math. Anal. Appl. 282 (2003), 256-265.

[CDC] M. CAVALCANTI and V. DOMINGOS CAVALCANTI, Global existence and uniform decay for the coupled Klein-Gordon-Schrödinger equations, NoDEA, Nonlinear differ. equ. appl. 7 (2000), 285-307.

[CaOq] M. M. CAVALCANTI and H. P. OQUENDO, Frictional versus viscoelastic damping in a semilinear wave equation. SIAM J. Control Optim. 42(4) (2003), 1310-1324

[FT1] I. FUKUDA and M. TSUTSUMI, On coupled Klein-GordonSchrödinger equations I, Bull. Sci. Engrg. Res. Lab. Waseda Univ. 69 (1975), 51-62.

[FT2] I. FUKUDA and M. TSUTSUMI, On coupled Klein-GordonSchrödinger equations II, J. Math. Analysis Applic. 66 (1978), 358-378. 
[FT3] I. FUKUDA and M. TSUTSUMI, On coupled Klein-Gordon Schrödinger equations III-Higher order interaction, decay and blowup, Math. Japonica 24 (1979), 307-321.

[FT4] I. FUKUDA and M. TSUTSUMI, On the Yukawa-coupled KleinGordon-Schödinger equations in three space dimensions, Proc. Japan Acad. 51 (1975), 402-405.

[Ha] A. HARAUX, Semi-groupes linaires et quations d'volution linaires priodiques. Universit Pierre et Marie Curie, Laboratoire d'Analyse Numrique. Prplication 78011 (1978)

[HaVo] N. HAYASHI and W. VON WAHL, On the global strong solutions of coupled Klein-Gordon-Schrödinger equations, J. Math. Soc. Japan 39 (1987), 489-497.

[Ho] L. F. HO, Observabilit frontire de l'quation des ondes, C. R. Acad. Sci. Paris Sr. I Math. 302, 443-446.

[K] V. KOMORNIK, Exact Controllability and Stabilization. The Multiplier Method, Mason- John Wiley, Paris, 1994.

[Lag] J. LAGNESE, Decay of solutions of wave equations in a bounded region with boundary dissipation. J. Diff. Equations 50 (1983), 163-182.

[LaWa1] H. LANGE and B. WANG, Regularity of the global attractor for the Klein-Gordon-Schrödinger equation, Math. Methods Appl. Sci. 22(17) (1999), 1535-1554.

[LaWa2] H. LANGE and B. WANG, Attractors for the Klein-GordonSchrödinger equation, J. Math. Phys. 40(5) (1999), 2445-2457.

[LaTa] I. LASIECKA and D. TATARU, Uniform boundary stabilization of semilinear wave equation with nonlinear boundary dissipation, Diff. and Integral Equations 6 (1993), 507-533.

[Li] J. L. LIONS, Quelques Métodes de Résolution des Problèmes Aux Limites Non Linéaires, Dunod, Paris, 1969.

[Li2] J. L. LIONS, Controlabilité exacte, perturbations et Stabilisation de systèmes distribués, Tome 1, Masson, Paris, (1988).

[LiMa] J. L. LIONS-E. MAGENES, Problèmes Aux Limites Non Homogènes et Applications, Dunod, Paris, 1968, Vol. 1.

[MoGoHa] A. MOSTAFA, O. GOUBET and A. HAKIM, Regularity of the attractor for a coupled Klein-Gordon-Schrödinger system, Diff. Integal Equ. 16(5) (2003), 573-581. 
[Oh] M. OHTA, Stability of Stationary States for the Coupled KleinGordon-Schrödinger Equations, Nonlinear Analysis, Theory, Methods and Appl. 27(4) (1996), 455-461.

GaDa] P. GAO and ZHENGDE DAI, Exponential attractor for dissipative Kein-Gordon-Schrödinger equations in $\mathbf{R}^{\mathbf{3}}$, Chim. Ann. Math. Ser. A. 21(2) (2000), 241-250.

[Yu] H. YUKAWA, On the interaction of elementary particles I, Proc. Physico-Math. Soc. Japan 17 (1935), 48-57.

[Zua] E. ZUAZUA, Exponential decay for the semilinear wave equation with locally distributed damping, Commun. Partial Differential Equations 15(2) (1990), 205-235.

Received 15 May 2006; accepted 13 November 2006

To access this journal online: http://www.birkhauser.ch 